MATEMATIKA, 2017, Volume 33, Number 1, 97-103

(C) Penerbit UTM Press. All rights reserved

\title{
Characterization of a hyperperfect group
}

\author{
${ }^{1}$ Saugata Purkayastha, ${ }^{2}$ Jituparna Goswami and ${ }^{3}$ Bhargab Debnath \\ ${ }_{1,2,3}$ Department of Applied Sciences(Mathematical Science Division) \\ Gauhati University, Guwahati-14, Assam, India \\ e-mail: ${ }^{1}$ sau.pur@rediffmail.com, ${ }^{2}$ jituparnagoswami18@gmail.com (corresponding author) \\ ${ }^{3}$ bhargabdnath14@rediffmail.com
}

\begin{abstract}
In this work, we have introduced the notion of hyperperfect group. A group of order $n$ is said to be hyperperfect if there exists a natural number $k$ such that $n-1=k[\sigma(n)-n-1]$, where $\sigma(n)$ denotes the sum of positive divisors of $n$. We have also established a condition under which a cyclic group is hyperperfect. We have established that no group of prime order is hyperperfect and investigated the same for groups of various non-prime order. We have also determined an upper bound of the order of a hyperperfect group.
\end{abstract}

Keywords Perfect number; hyperperfect number; cyclic group, upper bound.

2010 Mathematics Subject Classification 20D60.

\section{Introduction}

A natural number $n$ is called a perfect number if $n$ is equal to the sum of all its positive divisors, excluding $n$ itself. For example, 6 is perfect, since $6=1+2+3$. Since its inception, the notion of perfect number has been the center of attention for many mathematicians and the same has been studied in tandem with various other concepts from time to time. For instance, Leinster [1] extended the study of perfect numbers to finite groups. He defined a finite group to be perfect (or Leinster) if its order is equal to the sum of the orders of all proper normal subgroups of the group. As an interesting consequence of this approach, one may observe that a finite cyclic group is a Leinster group if and only if its order is a perfect number. The connection between perfect numbers and finite groups have also been studied by Medts [2]. In spite of all the endeavours made so far, a natural question that still remains open is whether there are odd perfect numbers or not.

As an obvious generalization of perfect numbers, the notion of hyperperfect numbers have been introduced in [3]. A natural number $n$ is called a a $k$-hyperperfect number if there exist an integer $k$ such that

$$
\sigma(n)=\frac{k+1}{k} n+\frac{k-1}{k},
$$

where $\sigma(n)$ is the sum of the positive divisors of $n$. It is often referred to as a hyperperfect number for simplicity. Clearly, a number is perfect if and only if it is 1-hyperperfect. Thus, for example, 6 is hyperperfect, since

$$
\sigma(6)=\frac{k+1}{k} 6+\frac{k-1}{k} \Rightarrow k=1 .
$$


Based on Leinster's concept to extend the study from perfect number to finite group, in this work we have tried to extend our study from hyperperfect number to finite group and such a group is termed as hyperperfect goup.

All the algebraic and number theoretic contents of this work can be found in [4] and [5] respectively.

\section{Hyperperfect group and its basic properties}

In this section we define hyperperfect group and investigate its basic properties.

Definition 2.1 A group of order $n$ is said to be hyperperfect if there exists a natural number $k$ such that

$$
n-1=k[\sigma(n)-n-1],
$$

where $\sigma(n)$ denotes the sum of positive divisors of $n$.

Example 2.1. Let $G=U(18)=\{1,5,7,11,13,17\}$. Here $o(G)=n=6$ and $\sigma(n)=\sigma(6)=$ 12. Since

$$
\begin{aligned}
& 6-1=k[12-6-1] \\
\Rightarrow & 5=5 k \\
\Rightarrow & k=1 \in \mathbb{N}
\end{aligned}
$$

so, $G$ is hyperperfect.

Example 2.2. Let $G=\mathbb{Z}_{10}=\{0,1,2,3,4,5,6,7,8,9\}$. Here, $o(G)=n=10$ and $\sigma(n)=\sigma(10)=18$. Since,

$$
\begin{aligned}
& 10-1=k[18-10-1] \\
\Rightarrow & 9=7 k \\
\Rightarrow & k=\frac{9}{7} \notin \mathbb{N}
\end{aligned}
$$

so, $G$ is not hyperperfect.

Theorem 2.1 Let $G$ be a cyclic group. Then $G$ is hyperperfect if and only if $n=k \epsilon(n)+1$, where $o(G)=n, k \in \mathbb{N}$ is a natural number and $\epsilon(n)$ stands for the sum of the orders of proper subgroups of $G$.

Proof Let $G$ be hyperperfect. By Definition 2.1, then there exists $k \in \mathbb{N}$, such that

$$
n-1=k[\sigma(n)-n-1] .
$$

Since $G$ is cyclic, therefore for each positive divisor $d$ of $n, \exists$ a subgroup of $G$ having order $d$. Then,

$$
\begin{aligned}
& \sigma(n)=\text { Sum of the orders of all subgroup of } G \\
& \sigma(n)-n-1=\text { Sum of the orders of all proper subgroup of } G=\epsilon(n) .
\end{aligned}
$$


Hence, $n=1+k \epsilon(n)$.

Conversely, let $n=1+k \epsilon(n)$. As $G$ is cyclic, we have $\epsilon(n)=\sigma(n)-n-1$. Thus, $n-1=k[\sigma(n)-n-1]$ and hence $G$ is hyperperfect.

Corollary 2.2 No prime order group is hyperperfect.

Proof We know that a prime order group is cyclic. Let $o(G)=n$, where $n$ is prime. This means that $G$ has no proper subgroup and thus $\sigma(n)=n+1$. If $G$ is hyperperfect then,

$$
\begin{aligned}
n & =1+k \epsilon(n)=1+k \cdot 0 \\
& =1, \text { which is not possible. }
\end{aligned}
$$

Theorem 2.3 No cyclic group of order $p^{t}$ is hyperperfect, where $p$ is a prime number and $t$ is a positive integer such that $t>1$.

Proof Let $G$ be a cyclic group of order $n=p^{t}$. If $G$ is hyperperfect, then by Theorem 2.1

$$
n=1+k \epsilon(n) \Rightarrow k=\frac{n-1}{\epsilon(n)}
$$

where $\epsilon(n)$ is the sum of the orders of all proper subgroup of $G$

$$
\begin{aligned}
k & =\frac{p^{t}-1}{p+p^{2}+p^{3}+\cdots+p^{t-1}} \\
& =\left(p^{t}-1\right) /\left(\frac{p\left(p^{t-1}-1\right)}{p-1}\right)=\frac{\left(p^{t}-1\right)(p-1)}{p^{t}-p} \\
& =\frac{p^{t+1}-p^{t}-p+1}{p^{t}-p}=p-1+\frac{p^{2}-2 p+1}{p^{t}-p} \\
& =p-1+\frac{p}{p^{t-1}-1}-\frac{2}{p^{t-1}-1}+\frac{1}{p^{t}-p} \notin \mathbb{N} .
\end{aligned}
$$

This shows that $G$ cannot be hyperperfect.

Corollary 2.4 No cyclic group of order $p^{2}$ is hyperperfect, where $p$ is a prime number.

Theorem 2.5 A cyclic group of order $p q$ is hyperperfect if and only if $p^{2} \equiv-1(\bmod (p+q))$, where $p$ and $q$ are distinct prime numbers.

Proof Let $G$ be a cyclic group of order $n=p q$ with $\operatorname{gcd}(p, q)=1$. Let $G$ be hyperperfect. Then from Theorem 2.1, we get

$$
k=\frac{n-1}{\epsilon(n)}
$$

where $\epsilon(n)$ is the sum of the orders of all proper subgroup of $G$ and $k \in \mathbb{N}$. Now,

$$
\begin{aligned}
k & =\frac{p q-1}{\epsilon(p q)}=\frac{p q-1}{p+q} \\
& =\frac{p(p+q)-\left(p^{2}+1\right)}{p+q}=p-\frac{p^{2}+1}{p+q} .
\end{aligned}
$$


Since $k$ is a natural number so, $\frac{p^{2}+1}{p+q}$ must be a natural number less than $p$. Thus,

$$
(p+q) \mid\left(p^{2}+1\right) \Rightarrow p^{2} \equiv-1(\bmod (p+q)) .
$$

Conversely, let

$$
\begin{aligned}
p^{2} & \equiv-1(\bmod (p+q)) \\
\Rightarrow & (p+q) \mid\left(p^{2}+1\right) \Rightarrow \frac{p^{2}+1}{p+q}=k_{1} \in \mathbb{N} .
\end{aligned}
$$

We must have $\frac{p^{2}+1}{p+q}<p$, since if $\frac{p^{2}+1}{p+q}>p$, then

$$
\begin{aligned}
& \frac{p^{2}+1}{p+q}>p \Rightarrow p^{2}+1>p^{2}+p q \\
\Rightarrow & p q<1, \text { which is absurd. }
\end{aligned}
$$

So, $p-\frac{p^{2}+1}{p+q}=k \in \mathbb{N}$, where, $k=p-k_{1}$. Now,

$$
\begin{aligned}
& p-\frac{p^{2}+1}{p+q}=k \\
\Rightarrow & \frac{p(p+q)-\left(p^{2}+1\right)}{p+q}=k \\
\Rightarrow & \frac{p^{2}+p q-p^{2}-1}{p+q}=k \\
\Rightarrow & \frac{p q-1}{p+q}=k
\end{aligned}
$$

or $n=1+k \epsilon(n)$.

Corollary 2.6 No cyclic group is hyperperfect whose orders are of the form $5 p, 11 p, 19 p$, $29 p, 31 p, 37 p, 47 p$ where $p$ is a prime number.

Theorem 2.7 Let $q=p(p-1)+1$ be a prime for prime $p$. Then a cyclic group $G$ of order $p q$ is hyperperfect.

Proof Given, $q=p(p-1)+1$ and $p, q$ are distinct primes. By Theorem 2.5, we know that if $G$ is cyclic of order $p q$ then $G$ is hyperperfect iff $p^{2} \equiv-1(\bmod (p+q))$, that is, it suffices to show that $p^{2} \equiv-1(\bmod (p+q))$. Now,

$$
\begin{aligned}
& q=p(p-1)+1 \\
\Leftrightarrow & q=p^{2}-p+1 \\
\Leftrightarrow & p+q=p^{2}+1 \\
\Leftrightarrow & p^{2}+1=p+q \\
\Leftrightarrow & p^{2}+1 \equiv 0(\bmod (p+q)) \\
\Leftrightarrow & p^{2} \equiv-1(\bmod (p+q)) .
\end{aligned}
$$


Theorem 2.8 If $k>0$ and $p=k+1$ is prime, then $\forall i>1$, such that $q=p^{i}-p+1$ is prime then a cyclic group of order $n\left(=p^{i-1} q\right)$ is hyperperfect.

Proof From Definition 2.1, we get

$$
\begin{aligned}
k & =\frac{n-1}{\sigma(n)-n-1} \\
& =\frac{p^{i-1} q-1}{\sigma\left(p^{i-1} q\right)-p^{i-1} q-1}
\end{aligned}
$$

where $n=o(G)$ and $k \in \mathbb{N}$ and

$$
\begin{aligned}
\sigma\left(p^{i-1} q\right) & =\sigma\left(p^{i-1}\right) \sigma(q) \\
& =\left(\frac{p^{i}-1}{p-1}\right)(q+1) \\
& =\frac{p^{i} q+p^{i}-q-1}{p-1} .
\end{aligned}
$$

Therefore

$$
\begin{aligned}
\sigma\left(p^{i-1} q\right)-p^{i-1} q-1 & =\frac{p^{i} q+p^{i}-q-1}{p-1}-p^{i-1} q-1 \\
& =\frac{p^{i} q+p^{i}-q-1-p^{i} q-p+p^{i-1} q+1}{p-1} \\
& =\frac{p^{i}-q-p+p^{i-1} q}{p-1}=\frac{p\left(p^{i-1}-1\right)+q\left(p^{i-1}-1\right)}{p-1} \\
& =\frac{\left(p^{i-1}-1\right)(p+q)}{p-1} \\
& =\frac{\left(p^{i-1}-1\right)\left(p+p^{i}-p+1\right)}{p-1} \quad\left[\because q=p^{i}-p+1\right] \\
& =\frac{\left(p^{i-1}-1\right)\left(p^{i}+1\right)}{p-1} .
\end{aligned}
$$

Again,

$$
\begin{aligned}
p^{i-1} q-1 & =p^{i-1}\left(p^{i}-p+1\right)-1 \quad\left[\because q=p^{i}-p+1\right] \\
& =p^{2 i-1}-p^{i}+p^{i-1}-1 . \\
\therefore k & =\left(p^{2 i-1}-p^{i}+p^{i-1}-1\right) /\left(\frac{\left(p^{i-1}-1\right)\left(p^{i}+1\right)}{p-1}\right) \\
& =\frac{(p-1)\left(p^{2 i-1}-p^{i}+p^{i-1}-1\right)}{\left(p^{i-1}-1\right)\left(p^{i}+1\right)} \\
& =\frac{p^{2 i}-p^{i+1}+2 p^{i}-p^{2 i-1}-p^{i-1}-p+1}{p^{2 i-1}-p^{i}+p^{i-1}-1} \\
& =p-1 \in \mathbb{N} \\
\therefore k & =p-1 \\
\Rightarrow p & =k+1 .
\end{aligned}
$$




\section{Upper bound for the order of a hyperperfect group}

In this section, we investigate for an upper bound for the order of a hyperperfect group. Let $G$ be a finite group and $o(G)$ be the order of $G$. We define

$$
\begin{aligned}
& D(G)=\text { Sum of orders of all subgroup of } G \\
& D^{*}(G)=\text { Sum of orders of all proper subgroup of } G \text { including }\{e\}
\end{aligned}
$$

and

$$
\begin{aligned}
& \delta(G)=\frac{D(G)}{o(G)} \\
& \delta^{*}(G)=\frac{D^{*}(G)}{o(G)} .
\end{aligned}
$$

Thus, if $o(G)=n$, then

$$
\begin{aligned}
& \delta(G)=\frac{D(G)}{n} \\
& \delta^{*}(G)=\frac{D^{*}(G)}{n} .
\end{aligned}
$$

Theorem 3.1 If $G$ is cyclic and hyperperfect with $o(G)=n$, then

$$
n<\sigma(n)\left(\frac{1+k \epsilon(n)}{1+\epsilon(n)}\right)
$$

Proof Let $G$ be cyclic, then

$$
\begin{aligned}
& D(G)=\sigma(|G|)=\sigma(n) \\
& D^{*}(G)=\epsilon(|G|)+1=\epsilon(n)+1
\end{aligned}
$$

By definition, we have

$$
\begin{aligned}
& D^{*}(G)<D(G) \Rightarrow \epsilon(n)+1<\sigma(n) \\
& \Rightarrow \frac{\epsilon(n)+1}{n}<\frac{\sigma(n)}{n} \Rightarrow \frac{n}{\epsilon(n)+1}>\frac{n}{\sigma(n)} .
\end{aligned}
$$

Since $G$ is hyperperfect, by Theorem 2.1 , we have

$$
\frac{1+k \epsilon(n)}{\epsilon(n)+1}>\frac{n}{\sigma(n)} \Rightarrow n<\sigma(n)\left(\frac{1+k \epsilon(n)}{1+\epsilon(n)}\right) .
$$

Corollary 3.2 If $n$ is perfect then $n<\sigma(n)$.

Proof Since a hyperperfect number is perfect if and ony if $k=1$ (see [3]) the result follows from Theorem 3.1 and for $k=1$. 


\section{Conclusion}

We have seen that Theorem 2.8 gives a characterization of a cyclic group of order $p q$, $\operatorname{gcd}(p, q)=1$ to be hyperperfect and conversely. Theorem 3.1 gives an upper bound of a hyperperfect number. Finally, Corollary 3.2 shows that if $n$ is perfect then $n<\sigma(n)$, which is in accordance with the fact that for $n$ perfect, $\sigma(n)=2 n$.

\section{References}

[1] Leinster, T. Perfect Numbers and Groups. arXiv:math/0104012v1[math.GR]. 1st April 2001.

[2] Medts, T. D. and Maroti, A. Perfect numbers and finite groups, rendiconti del seminario matematico della Universita di Padova. The Mathematical Journal of the University of Padua. 2013. 129: 17-33.

[3] McCranie, J. S. A study of hyperperfect numbers. Journal of Integer Sequences. 2000. 3 , Article 00.1.3.

[4] Gallian, J. A. Contemporary Abstract Algebra. 4th Edition. Narosa Publishing House Pvt. Ltd. 1999.

[5] Burton, D. M. Elementary Number Theory. 6th Edition. Tata McGraw Hill Education Pvt. Ltd. 2010. 